exist given Blackburn's history, he should be invited for an interview and the IACUC should meet him with an open mind and without accusation. Within this interview, several things should be confirmed such as he truly meets the requirements of a nonaffiliated member as per PHS policy and guidance ${ }^{1,2}$. During the interview, committee members' concerns could be incorporated into appropriate questions. It would be important to ascertain if Blackburn fully understands the intended role, responsibilities and confidentiality of an IACUC member, which should be a standard practice with any potential new committee member. Blackburn should also be asked why he would like to be part of the IACUC and how he feels about the use of laboratory animals in the biomedical field.
If he strongly opposes the use of animals entirely, he may no longer want to take the position or the IACUC may decide that he is not a suitable candidate due to the potential of strong bias. In addition, opinions from the senior administration and the principle investigators should be obtained before making any decision.

It is important to have a balanced IACUC that includes members who can constructively criticize protocol applications without impeding the overall efficiency and effectiveness of the committee. Hence, after additional information is confirmed, Blackburn may be considered as a nonaffiliated member in the IACUC. If accepted, providing deadlines to reviewers and following a strict schedule for meetings would help eliminate concerns for 'slowing down' the work of the committee.

Lisa Quinn and Damodaran Annamalai* Division of Comparative medicine, Massachusetts Institute of Technology, Cambridge, MA, USA.

*e-mail: annamald@mit.edu

Published online: 21 May 2019

https://doi.org/10.1038/s41684-019-0307-9

\section{References}

1. National Institutes of Health. NOT-OD-15-109: Guidance on Qualifications of IACUC Nonscientific and Nonaffiliated Members. National Institutes of Health Office of Extramural Research. https://grants.nih.gov/grants/guide/notice-files/NOTOD-15-109.html

2. U.S. Department of Health and Human Services. Public Health Service Policy on Human Care and Use of Laboratory Animals (US Department of Health and Human Services: Washington, DC, 1986. revised 2015).

\title{
Potential value, but proper vetting required
}

A challenge inherent to the management of an animal care and use program (ACUP) is the protection of the integrity of the program and preservation of the overall mission to ensure that animal research activities promote "human or animal health, the advancement of knowledge, or the good

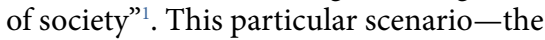
appointment of a nonaffiliated IACUC member with a past that may suggest an opposition to the use of animals in biomedical research-depicts such a challenge.

Given the Compliance Director has ostensibly qualified Mr. Blackburn as a potential nonaffiliated member per the PHS Policy ${ }^{2}$ and USDA Animal Welfare Regulations ${ }^{3}$, Great Eastern University (GEU) must now consider whether Blackburn's participation would enhance and strengthen committee functions. As a nonaffiliated member, Blackburn would review proposed animal activities and guidance documents used to manage and implement the GEU ACUP. If he can conduct these evaluations objectively, his contributions could ensure the presence of a 'public conscience' in these processes ${ }^{4}$. The Compliance Director and Covelli hypothesize that Blackburn thus has the potential to add value to the Committee.

GEU must first verify that Blackburn has no immediate familial relationships or other discernable ties to the university. The PHS Frequently Asked Questions ${ }^{5}$ state that "real or perceived conflicts of interest must be avoided to ensure the IACUC's and the institution's integrity." For instance, it would be concerning if his voting records or fundraising history indicated ties to animal activist organizations. As part of his appointment, Blackburn would be expected to thoroughly examine whether he has any relationships-personal or business - that would not permit him to contribute with impartiality.

Considering Blackburn's persistent attention to the use of animals in biomedical research, it is appropriate for the IACUC members to question whether Blackburn would be capable of representing the general community's interests and making unbiased decisions about the use of animals in proposed research activities. His apparent perspective on animal research is likely more in line with the American public's views than those of working researchers; roughly half of U.S. adults hold an oppositional view ${ }^{6}$. However, the public tends to be poorly informed about the level of care research animals receive, the scrutiny projects are subjected to prior to approval, and the continued efforts to replace, reduce, and refine. A naivety about the oversight and welfare considerations in animal research and a solidified stance against animals in research may appear the same at first glance. A review of his positions as senator may indicate whether Blackburn's intention was to eliminate animal research entirely or require stronger oversight.

The next step is to invite Blackburn for an interview with the IACUC. A tour of facilities and a brief training on the regulations may give insight into how
Blackburn would approach the role of an IACUC member. If it appears that Blackburn's goal was not to eliminate animal use, but rather help to ensure and support the idea that animals should be used judiciously and only as necessary, then his ideas are consistent with the general philosophy of the IACUC. Blackburn may become an excellent nonaffiliated member and addition to Great Eastern University.

\section{Jessi Kittel* and William Greer \\ University of Michigan, Ann Arbor, USA. \\ *e-mail: jessik@med.umich.edu}

Published online: 21 May 2019

https://doi.org/10.1038/s41684-019-0308-8

\footnotetext{
References

1. Institute for Laboratory Animal Research. Guide for the Care and Use of Laboratory Animals 8th edn. Appendix B. U.S Government Principles for the Utilization and Care of Vertebrate Animals Used in Testing, Research, and Training (National Academies Press, Washington, DC, 2011). https://www.ncbi.nlm.nih.gov/books/NBK54048/

2. U.S. Department of Health and Human Services. Public Health Service Policy on Human Care and Use of Laboratory Animals. PHS Policy IV.A.3.b.(4) (2015). https://olaw.nih.gov/policies-laws/ phs-policy.htm\#AnimalWelfareAssurance

3. Animal Welfare Regulations Section 2.31 (a) and (b) https://www. aphis.usda.gov/animal_welfare/downloads/AC_BlueBook_AWA FINAL_2017_508comp.pdf

4. Office of Laboratory Animal Welfare, National Institutes of Health. Institutional Animal Care and Use Committee Guidebook, 2nd edn. (2002) https://grants.nih.gov/grants/olaw/guidebook.pdf . Office of Laboratory Animal Welfare, National Institutes of Health. PHS Policy on the Humane Care and Use of Laboratory Animals: Frequency Asked Questions https://olaw.nih.gov/ guidance/faqs

6. Pew Research Center. Americans, Politics, and Science Issues: Opinion About the Use of Animals in Research (2015) http://www. pewresearch.org/science/2015/07/01/chapter-7-opinion-aboutthe-use-of-animals-in-research/
} 